\title{
Using a form-finding model to analyze the effect of actin bundles on the stiffness of a cytoskeleton network ${ }^{*}$
}

\author{
Bao-long LI, Yi-fan WANG, Jing-hai GONG ${ }^{\dagger+}$ \\ (Department of Civil Engineering, Shanghai Jiao Tong University, Shanghai 200240, China) \\ †E-mail: gongjh@sjtu.edu.cn \\ Received Nov. 11, 2013; Revision accepted Apr. 16, 2014; Crosschecked Aug. 25, 2014
}

\begin{abstract}
Networks of actin filaments and bundles are ubiquitous in cellular cytoskeletons, but the elasticity of the network is not well understood. In this paper, a computational model based on form-finding analysis is proposed to investigate the stiffness of cytoskeleton networks consisting of actin filaments and bundles. The model shows that networks with parallel bundles aligned in the stretching direction are stiffer than those with randomly distributed bundles. The results provide a mechanical explanation for the experimental observation that cells primarily create parallel rather than disordered bundles during cell adhesion and cell motion. The effect of filament undulations on network stiffness is explored briefly. The results show that undulations can soften the network by increasing the bending-dominated deformations in filaments and bundles. Finally, we find that the effect of the relative density of bundles depends on their orientation. Increasing the density of randomly distributed bundles has no effect on the stiffness of cells, but softens the cytoskeleton network. In contrast, the stiffness of networks of parallel bundles first increases, then reduces as the relative density of bundles increases. The stiffest network is a mixture of actin filaments and bundles.
\end{abstract}

Key words: Cytoskeleton network, Form-finding model, Elastic modulus, Actin bundles doi: $10.1631 /$ jzus.A13b0287

Document code: A

CLC number: Q66

\section{Introduction}

In eukaryotic cells, the actin cytoskeleton network plays an important role in the maintenance of cellular architecture and numerous physical processes, including cell adhesion, cell migration, and cell division (Ethier and Simmons, 2007). The mechanical properties of these actin cytoskeleton networks are critical in determining how forces are generated and transmitted in living cells (Gardel et al., 2004).

The actin cytoskeleton is a composite intracellular biopolymer network, usually composed of actin filaments and bundles cross-linked by actin-binding proteins (Fig. 1). Actin filament bundles are highorder cytoskeletal structures made of actin filaments

\footnotetext{
"Corresponding author

* Project supported by the Shanghai Jiao Tong University-Johns Hopkins University International Cooperation Project (No. TS0520101002)

(C) Zhejiang University and Springer-Verlag Berlin Heidelberg 2014
}

packed together in concert with specific actin-binding proteins, like fascin and $\alpha$-actinin (Tseng et al., 2005). In eukaryotic cells, actin filament bundle structures are common and include stress fibers, which are long and straight and align primarily in parallel with the long axis of the cell (Stricker et al., 2010). Shin et al . (2004) reported that an actin bundle has a similar elastic modulus to that of a single actin filament, and an average bending stiffness more than two orders of magnitude larger than that of a bundle of uncrosslinked actin filaments. Therefore, the existence of actin bundles in cytoskeleton networks has a significant influence on the mechanical properties and performance of cells.

Recently, a number of computational models, designed to complement experimental work from the point of view of mathematics and mechanics, have been proposed to predict the elasticity of cytoskeleton networks. These models include the open-foam model (Satcher and Dewey, 1996), tensegrity model 


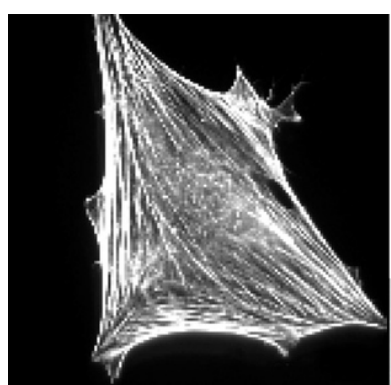

(a)

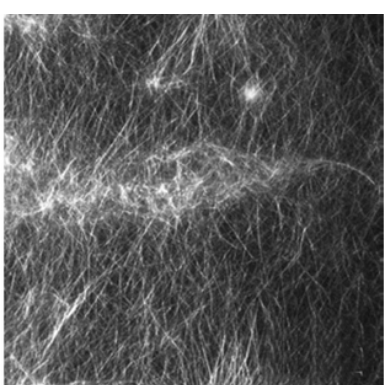

(b)
Fig. 1 A combination of orthogonal meshworks and bundles in cells and reconstituted F-actin networks microinjected with fascin and $\alpha$-actinin (Tseng et al., 2005)

(a) Fluorescence micrographs of cells injected with fascin and $\alpha$-actinin; (b) Confocal micrographs of actin filament networks in the presence of fascin and $\alpha$-actinin

(Stamenovic and Coughlin, 2000), cable network model (Coughlin and Stamenovic, 2003), and others (Stamenovic and Ingber, 2002; Lim et al., 2006). Although these models provide an insight into understanding the complicated cellular responses to an external mechanical stimulus, they have regular topologies and seldom consider the effects of variation in cytoskeletal constituents on the elasticity of networks. A 2D network model of cross-linked semiflexible filaments has been proposed (Onck et al., 2005), and extended to a 3D model (Huisman et al., 2007). The model explicitly accounts for filament properties and network architecture. However, it does not take into account the maximum axial capacity of filaments and the properties of cross-linkers. The latest cellular mechanical models, like the coarse grained model (Kang et al., 2011) and the Brownian dynamics simulation model (Kim et al., 2009a; $2009 \mathrm{~b}$ ), break through the limits of simple and regular topologies. These models have more complex and diverse topologies based on stochastic theory or Brownian dynamics, and consider in more detail the effects of cytoskeletal constituents on cellular mechanical properties. However, few of them concentrate on the performance of actin bundles in cytoskeleton networks under an external mechanical stimulus.

In this paper, we use a $2 \mathrm{D}$ form-finding model (Gong et al., 2013) to investigate the stiffness of a cytoskeleton network consisting of actin filaments and bundles. The elastic modulus of cytoskeleton networks with different alignments of actin bundles is computed to explain the experimental observation that actin bundles always align in parallel in living cells. In addition, the effect of the relative density of actin bundles on the stiffness of the cytoskeleton network is explored, and the influence of filament undulations is briefly taken into consideration.

\section{Form-finding model}

\subsection{Basic principle of the form-finding model}

First, a form-finding model is proposed to investigate the elasticity of a cytoskeleton network without actin bundles. The network consists of an actin network modeled as beams, which can bear tension, compression, bending, and torsion, crosslinked by actin-binding proteins modeled as cables, which can bear tension only. Either $\alpha$-actinin or filamin cross-linkers may be appropriate here, but the model parameters we used (Table 1) are essentially aligned with filamin properties. The process of formfinding is performed using nonlinear finite element method at large deformation. The dimensional and material properties for the network (Table 1) were selected from available experimental measurements.

In this study, we extend the form-finding model to build up a model of cytoskeletal structure composed of cross-linked networks of filaments and bundles. At this stage, our goal is to demonstrate the suitability of the form-finding method. Thus, we assumed that seven actin filaments form an actin bundle whose cross-section is round (Tseng et al., 2002). Therefore, the moment of inertia is calculated by the formula $I=\pi D^{4} / 64$, where $D$ is the diameter of the cross-section. The geometrical properties of an individual actin filament and a bundle are listed in Table 2 . Bundles are also modeled as beam elements in nonlinear finite element analysis. It is evident that an actin bundle has the same elastic modulus of $1.4 \mathrm{GPa}$ as an individual actin filament in this model, but the bending stiffness of an actin bundle is nearly $3^{4}=81$ times larger than that of seven unbundled actin filaments.

\subsection{Process of model construction}

The process of modeling was as follows:

Step 1: Define a square domain $(10 \mu \mathrm{m} \times 10 \mu \mathrm{m})$ as the extent of the initial cytoskeleton network. 
Step 2: Actin filaments and bundles are placed sequentially within the domain stochastically until

Table 1 Dimensions and material properties of actin filaments and cross-linkers

\begin{tabular}{|c|c|c|c|}
\hline \multicolumn{2}{|c|}{ Parameter } & Value & Ref \\
\hline \multicolumn{2}{|c|}{$\begin{array}{l}\text { Elastic modulus of actin } \\
\text { filaments }(\mathrm{GPa})\end{array}$} & 1.4 & $\begin{array}{l}\text { Matsushita } \\
\text { et al. }(2010)\end{array}$ \\
\hline \multicolumn{2}{|c|}{$\begin{array}{l}\text { Diameter of actin filaments } \\
\quad(\mathrm{nm})\end{array}$} & 7 & $\begin{array}{l}\text { Matsushita } \\
\text { et al. }(2010)\end{array}$ \\
\hline \multicolumn{2}{|c|}{$\begin{array}{l}\text { Length of actin filaments } \\
\qquad(\mu \mathrm{m})\end{array}$} & $5 \pm 2$ & $\begin{array}{l}\text { Kasza et al. } \\
\quad(2010)\end{array}$ \\
\hline \multicolumn{2}{|c|}{$\begin{array}{l}\text { Length of actin filament } \\
\quad \text { segments }^{1}(\mu \mathrm{m})\end{array}$} & $0.3 \pm 0.06$ & \\
\hline \multicolumn{2}{|c|}{$\begin{array}{l}\text { Relative density of actin } \\
\text { filaments }{ }^{2}(\%)\end{array}$} & $0.15-0.30$ & $\begin{array}{c}\text { Ethier and } \\
\text { Simmons (2007) }\end{array}$ \\
\hline \multicolumn{2}{|c|}{$\begin{array}{l}\text { Yield tensile force of actin } \\
\text { filaments }(\mathrm{nN})\end{array}$} & 0.25 & $\begin{array}{l}\text { Lin et al. } \\
(2010)\end{array}$ \\
\hline \multicolumn{2}{|c|}{$\begin{array}{l}\text { Maximum length of } \\
\text { cross-linkers }(\mu \mathrm{m})\end{array}$} & 0.3 & $\begin{array}{l}\text { Furuike et al. } \\
\qquad(2001)\end{array}$ \\
\hline \multicolumn{2}{|c|}{$\begin{array}{l}\text { Stiffness of cross-linkers } \\
\text { (EA) }(\mathrm{nN})\end{array}$} & 1.35 & $\begin{array}{l}\text { Furuike et al. } \\
\qquad(2001)\end{array}$ \\
\hline \multicolumn{2}{|c|}{$\begin{array}{c}\text { Yield tensile force of } \\
\text { cross-linkers }(\mathrm{pN})\end{array}$} & 60 & $\begin{array}{l}\text { Ferrer et al. } \\
\quad(2008)\end{array}$ \\
\hline \multicolumn{4}{|c|}{$\begin{array}{l}{ }^{1} \text { The spacing of periodic binding sites to connect the cross-linker } \\
\text { on an actin filament; }{ }^{2} \text { Relative density is the amount of acti } \\
\text { filaments per unit volume of filament network }\end{array}$} \\
\hline \multicolumn{4}{|c|}{$\begin{array}{l}\text { Table } 2 \text { Assumed geometrical properties of an indi- } \\
\text { vidual actin filament and an actin filament bundle in } \\
\text { this model }\end{array}$} \\
\hline Component & $\begin{array}{c}\text { Diameter } \\
(\mathrm{nm})\end{array}$ & $\begin{array}{c}\text { Cross-section } \\
\text { area }\left(\mathrm{nm}^{2}\right)\end{array}$ & $\begin{array}{r}\text { Moment of } \\
\text { inertia }\left(\mathrm{nm}^{4}\right) \\
\end{array}$ \\
\hline 1 actin filamen & 7 & 25 & 118 \\
\hline 1 actin bundle & 21 & 175 & 9546 \\
\hline
\end{tabular}

their relative densities reach the specified value. For each actin filament, the length is sampled from a truncated Gaussian distribution (negative values are ignored) with mean and standard deviation as shown in Table 1. The centroidal coordinates and angle of orientation of each actin filament are determined from uniform random distributions that cover the domain $(X: 0-10 \mu \mathrm{m}, Y: 0-10 \mu \mathrm{m}, \theta: 0-2 \pi)$. It is assumed that the length and centroidal coordinates of the actin bundle obey the same distribution as the actin filaments, but the angle of orientation of the actin bundle can be controlled to obey a uniform random distribution within a range of specified spread in bundle orientation.

Step 3: Actin-binding proteins (filamin) are modeled as cable element cross-linkers to connect the actin filaments and bundles into a network. Networks are generated in two steps: (1) actin filaments and bundles are divided into several segments by actinbinding sites, whose lengths are determined by the pore size in real cytoskeleton networks. (2) crosslinkers are generated by connecting any two actinbinding sites whose distance apart is less than the length of the maximum cross-linker (Table 1).

Step 4: After a model is created by steps 1 to 3 , a form-finding analysis is carried out to compute the final equilibrium shape of the actin network. The form-finding analysis is accomplished using nonlinear finite element analysis after a small tensile pre-stress force is applied to the cross-linkers. Iteration is then performed until a self-equilibrium configuration is achieved (Fig. 2).
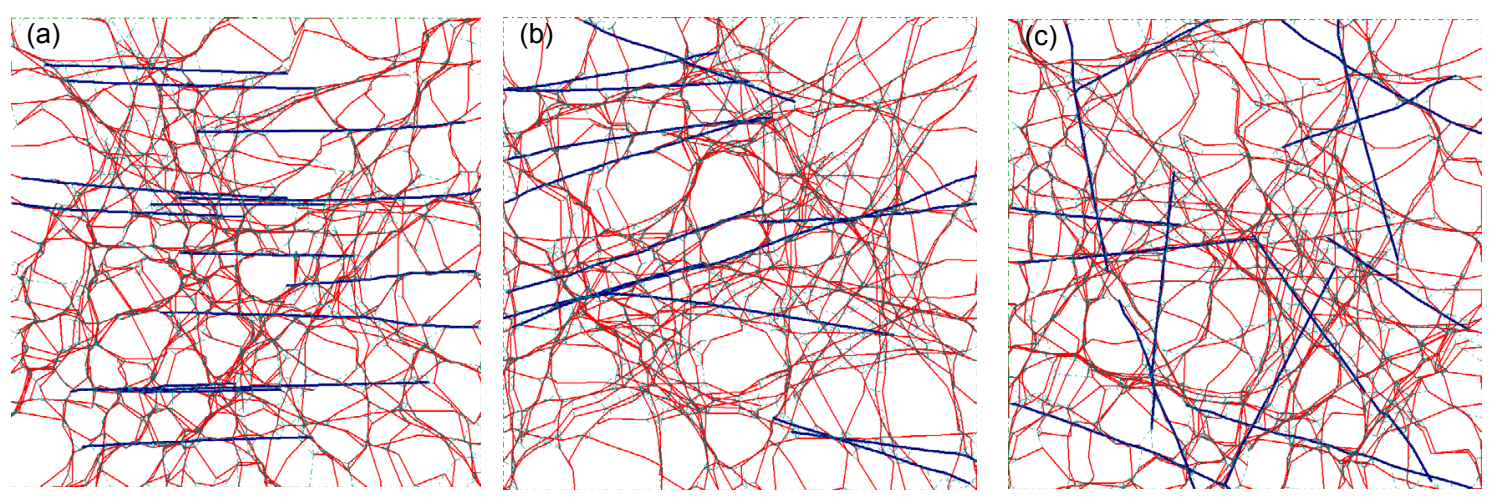

Fig. 2 Layouts of the form-finding model containing both filaments and bundles

The spread in bundle orientation in (a), (b), and (c) is $0^{\circ}, 20^{\circ}$, and $90^{\circ}$, respectively. The red line denotes actin filaments and the blue lines denote the bundles. For interpretation of the references to color in this figure, the reader is referred to the web version of this article 
The pre-stress force in cross-linkers is used to facilitate the form-finding analysis, and its magnitude can be calibrated by comparing the predicted filament undulations with experimental observations. A large pre-stress equates to a severe actin filament undulation and a small force to a gentle fluctuation. The maximum offset of all the actin-binding sites of filaments and bundles after form-finding, which reflects the undulation index of actin filament network samples, is set to $0.5 \mu \mathrm{m}$ unless otherwise specified. After form-finding, the elastic modulus $(E)$ of the network is determined by the results from a horizontal extension of the model. Given a finite stretch of $0.8 \mu \mathrm{m}$ (Gong et al., 2013), the effective engineering strain and stress can be calculated. The effective elastic modulus (actually, the secant modulus of actin networks with an overall strain of 0.08 ) is the stress divided by the strain. Note that an actin filament network with all bundles parallel or oriented within a particular angle in one direction will behave differently depending on the stretching direction. Just like anisotropic materials, the network stiffness differs along the stretching direction and the vertical direction. The elastic modulus in this study refers to cytoskeleton stiffness in the stretching direction only.

Since we are going to explore how the orientation of actin bundles in networks influences the stiffness of the cytoskeleton, the angle of orientation of the actin bundles needs to be adjustable. In this study, the parameter 'spread in bundle orientation' is defined as the absolute maximum value of the angle between the orientation of bundles and the horizontal direction in which the model is stretched. For instance, if the spread in bundle orientation is set to $20^{\circ}$ before modeling, each of the bundles to be generated in the model is sampled with an orientation angle to the horizontal direction from uniform random within $\left[-20^{\circ}, 20^{\circ}\right]$. Consequently, all the bundles will align in the horizontal direction if the spread in bundle orientation is equal to 0 . Furthermore, bundles have a uniform random distribution in every direction when the spread in bundle orientation is equal to $90^{\circ}$ (Fig. 2).

The total amount of actin filaments in a formfinding model is determined by the relative density of actin filaments (Table 1). In this study, the relative density of actin filaments is fixed at $0.3 \%$ throughout. Thus, the amount of actin filament material employed in each model is constant. Each actin filament in the model exists in the form of either individual filaments or a component of an actin bundle. The amount of bundles in a model is determined by the relative density of bundles, which is the ratio between the amount of filament material that is packed together to form bundles and the amount of total filament material. This parameter must be set before building up the cytoskeleton network model that consists of individual filaments and bundles. For instance, given a network model consisting of 700 actin filaments, if the relative density of bundles is $10 \%, 10 \% \times 700=70$ actin filaments are expended to generate 10 bundles and $700-70=630$ actin filaments remain in this network. The relative density of bundles can vary from $0 \%$ (no bundles in the model) to $100 \%$ (no individual filaments in the model). When the relative density of bundles is between $0 \%$ and $100 \%$, the network model consists of both individual filaments and filament bundles. The influence of the spread in bundle orientation, relative density of bundles and filament undulations on network stiffness was studied using sets of 100 samples (Gong et al., 2013) unless otherwise specified.

Compared with the aforementioned models, the form-finding model provides a means to generate realistic cytoskeletal network topologies incorporating major biological features. Another useful feature of the form-finding model is that it is inherently able to handle large deformations, since most biologically relevant deformations are large. Furthermore, the form-finding model captures the influential roles that actin filaments, actin-binding proteins, and actin bundles play in the stiffness of a cell.

\section{Results}

\subsection{Spread in bundle orientation to the stretching direction}

In living cells, actin bundles are critical to cell functions involving changes in cell shape, including migration, cell alignment, and cell contractility. Actin bundles are observed to align primarily in parallel with the long axis of cells, which is very different from a disordered filament network (Shin et al., 2004; Stricker et al., 2010). It seems that the alignment of actin bundles is of great importance to the mechanical properties of cells. In this section, the elastic modulus 
of cytoskeleton network models with different spreads in orientation of actin bundles is computed to investigate the reason why cells create parallel rather than disordered bundles, from the point of view of mechanics. The method for computing the elastic modulus of the form-finding model is described in Section 2.

The influence of the spread in bundle orientation to the stretching direction on network stiffness was studied using a sets of 1000 samples to obtain more reasonable curves (Fig. 4), with the spread in bundle orientation varied from $0^{\circ}$ to $90^{\circ}$ in increments of $10^{\circ}$. The relative densities of the bundles were $20 \%, 40 \%$, $60 \%$, and $80 \%$. The layouts of the selected samples are shown in Fig. 3. While actin filaments curved after form-finding analysis (Fig. 3), the bundles remained nearly straight because of their relative large bending stiffness. The variation in the predicted elastic modulus of networks with different spreads in the orientation of actin bundles is shown in Fig. 4. The spread in bundle orientation was negatively correlated to the stiffness of the network, and an approximately linear relation can be observed in each curve. The results suggest that parallel bundles aligned in the direction of an external force, the way in which actin bundles in living cells primarily exist, provide greater cell stiffness than randomly distributed bundles. Therefore, it is better for cells to generate parallel bundles than disordered bundles during cell adhesion and cell motion.

\subsection{Filament undulations soften the cytoskeleton network}

Filament undulations cause transverse bending in actin filaments and affect the stiffness of cytoskeleton networks (Onck et al., 2005). The pre-stress force applied to the cross-linkers in the form-finding model was positively correlated to the filament undulations. During the form-finding analysis, a large pre-stress force always results in a large offset of the actin-binding sites of actin filaments (these are segment ends described in Section 2). Therefore, the maximum offset (MO) of all the actin-binding sites of filaments and bundles can be used as an index to reflect the intensity of filament undulations.

In this study, the MO values changed from 0.1 to $0.6 \mu \mathrm{m}$, which represented the variation in filament undulations from gentle to relatively severe. We used

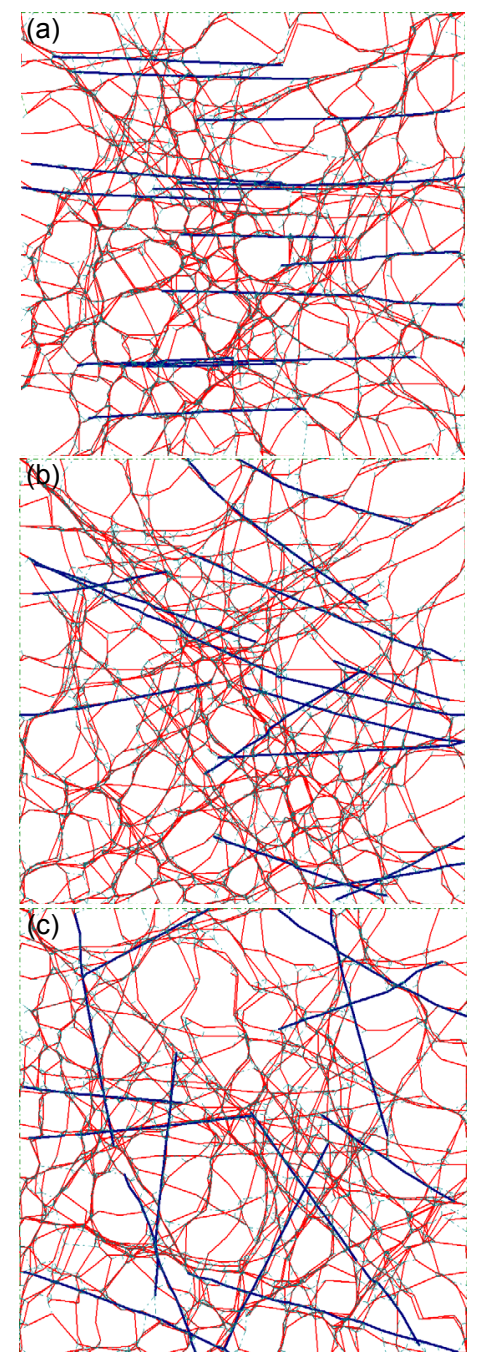

Fig. 3 Layouts of selected samples with different spreads in bundle orientation: (a) $0^{\circ}$, (b) $40^{\circ}$, and (c) $90^{\circ}$ The relative density of bundles was $40 \%$. The color lines are the same as that shown in Fig. 2

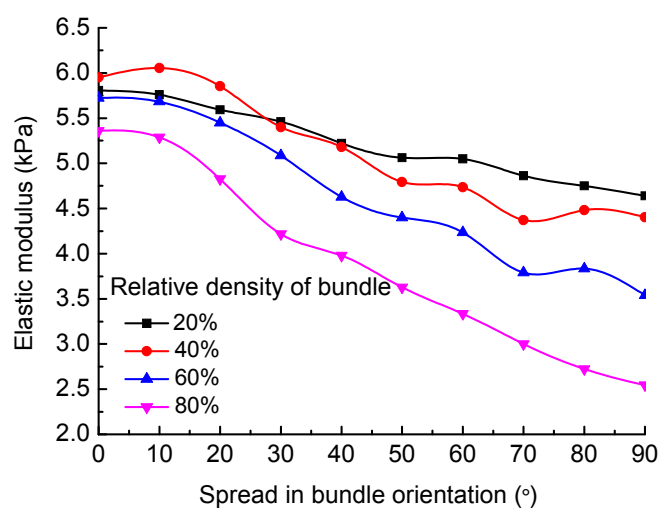

Fig. 4 Variation in average stiffness of networks with spread in bundle orientation

The size of each sample was 1000 
six sets of samples (100 in each set), across MO values ranging from 0.1 to $0.6 \mu \mathrm{m}$ in increments of $0.1 \mu \mathrm{m}$, to investigate briefly the effect of filament undulations on the elastic modulus of the cytoskeleton network. The same analysis was performed five times for different bundle relative densities of $0 \%$, $20 \%, 40 \%, 60 \%$, and $80 \%$, respectively. The spread in bundle orientation was set to 0 in each model. Fig. 5 clearly shows that a high intensity of filament undulations causes severe bending deformation in actin filaments. The variation in the average elastic modulus of networks with the filament undulations is shown in Fig. 6. Filament undulations can soften the stiffness of networks when the relative density of bundles is low. Thus, the stiffness of networks in which the relative density of bundles was $0 \%$ decreased the most. As the relative density of bundles increased, the softening effect of filament undulations weakened. The stiffness was almost unchanged when the relative density of bundles was $60 \%$, and increased slightly when it was $80 \%$.

\subsection{Relative density of bundles}

Considering the results in Section 3.1, the relative density of bundles might have distinct influences on the stiffness of cytoskeleton networks when the actin bundles align in parallel or distribute randomly. Although the bundles are observed primarily to align in parallel, a study of actin bundles distributed randomly is still useful to understand the organizational principles of filament networks. Therefore, in this section we study the effects of the relative density of bundles in one case in which most of the actin bundles align in parallel along the stretching direction, and in another case in which all of the bundles are distributed randomly in the network.

\section{Parallel bundles}

A group of 10 studies (100 samples in each study) was analyzed where the relative density of bundles varied from $0 \%$ to $90 \%$ in increments of $10 \%$. For each study, the elastic modulus of each sample was computed six times with different intensities of filament undulations, which were embodied in the MO values. Fig. 7 shows the layouts of selected samples, and the variation in network stiffness with the relative density of bundles is shown in Fig. 8. The curves in Fig. 8 display a trend in the relationship between network stiffness and bundle relative density. The

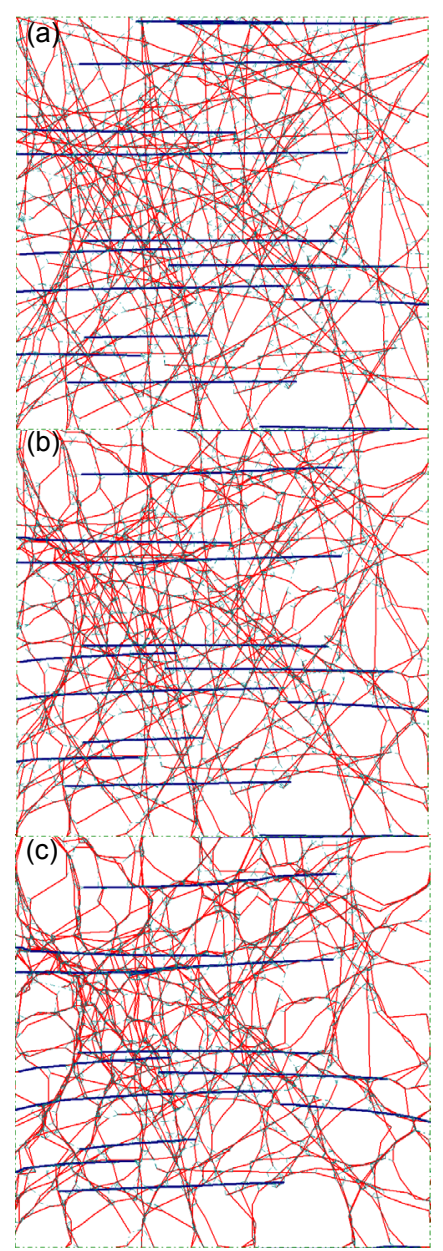

Fig. 5 Layouts of same samples with different maximum offset values of $0.1 \mu \mathrm{m}(\mathrm{a}), 0.3 \mu \mathrm{m}$ (b), and $0.6 \mu \mathrm{m}$ (c)

Filament undulations became more severe from (a) to (c), and as a result, more obvious bending occurred in the actin filaments. The relative density of bundles in each model was $40 \%$. The color lines are the same as that shown in Fig. 2

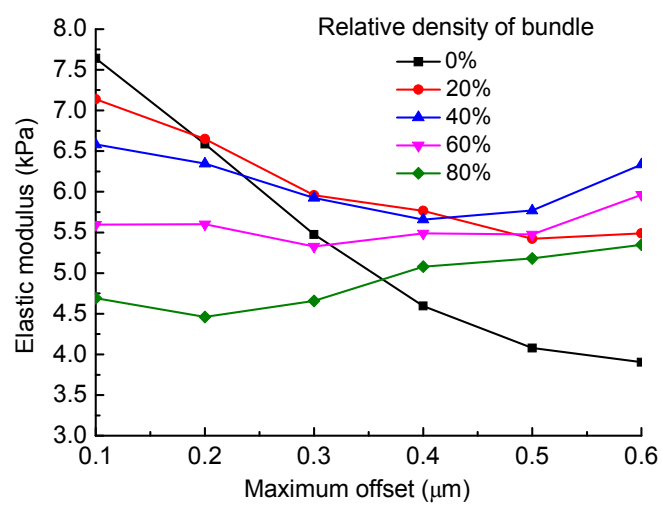

Fig. 6 Variation in average stiffness of networks with maximum offset values of from 0.1 to $0.6 \mu \mathrm{m}$ 


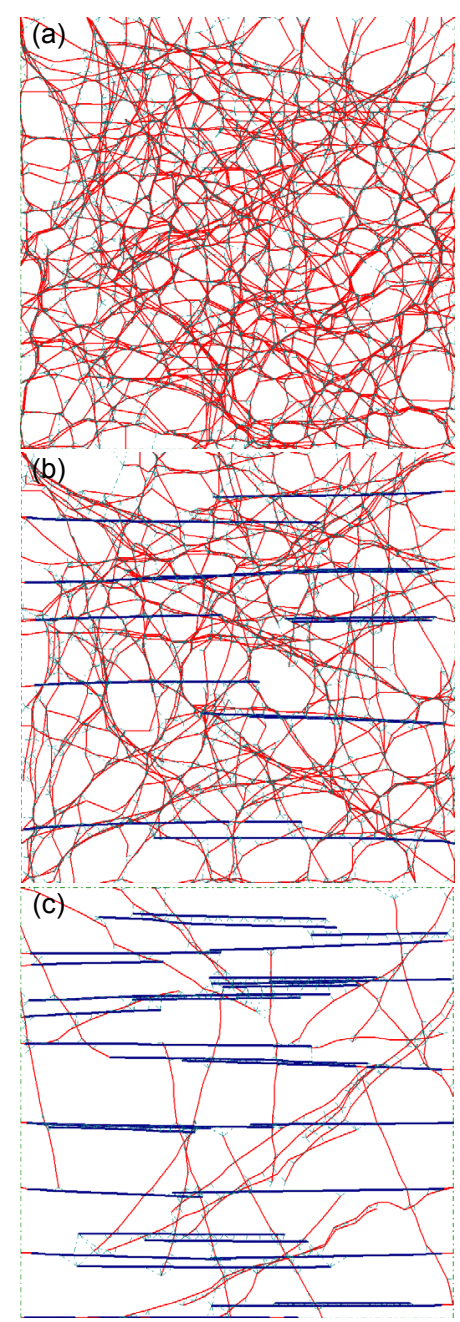

Fig. 7 Layouts of selected samples with different bundle relative densities of $0 \%(a), 40 \%$ (b), and $90 \%$ (c) The angle of all bundles was $0^{\circ}$, i.e., the bundles were aligned in parallel along the stretching direction. The maximum offset was $0.3 \mu \mathrm{m}$. The color lines are the same as that shown in Fig. 2

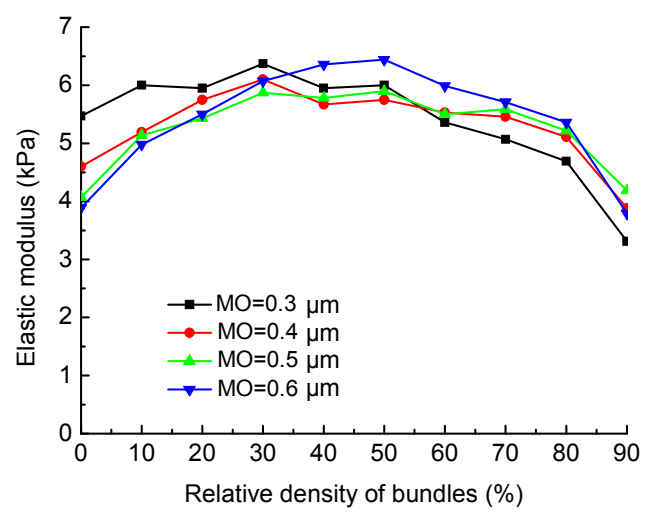

Fig. 8 Variation in the average stiffness of networks with the relative density of bundles under different values of maximum offset (MO)

The angle of all bundles was $0^{\circ}$ in each model average elastic modulus first increases and then decreases, when the MO value changes from 0.3 to 0.6 $\mu \mathrm{m}$. The results of this work indicate that more actin bundles do not improve the stiffness of cells when the amount of actin filament material is constant. The stiffest network is a mixture of actin filaments and bundles. Since the filament undulations observed were obvious (corresponding to an $\mathrm{MO}$ value greater than $0.3 \mu \mathrm{m}$ ), when the parallel bundles were aligned in the direction of stretching, the relative density of bundles in the stiffest network was in the range of $30 \%$ to $50 \%$.

\section{Randomly distributed bundles}

The same studies as above were reanalyzed for the case in which all bundles are distributed uniformly and randomly. Fig. 9 shows the layouts of selected samples, and the data from all the studies are provided in Fig. 10. Except for a slight increase appearing at low bundle relative densities, when the maximum offset was 0.5 or $0.6 \mu \mathrm{m}$, the average stiffness of the network decreased as the relative density of bundles changed from $0 \%$ to $90 \%$, regardless of whether the filament undulations were gentle or relatively severe. It seems that the disordered actin bundles make no contribution to increasing the stiffness of cells: on the contrary, they decrease the stiffness. It is an evolutionary advantage for cells to create parallel bundles rather than disordered bundles to facilitate cellular functions like immigration and cell motion. Another feature of the results shown in Fig. 8 is that when the bundle relative density is as high as $70 \%$, the average elastic modulus predicted for different MO values becomes similar because most actin filament material is packed into bundles that are not sensitive to filament undulations.

\section{Discussion}

Networks of cross-linked and bundled actin filaments are ubiquitous in cellular cytoskeletons, but knowledge of their mechanical properties is far from complete. In this study, we explored the stiffness of a cytoskeleton network consisting of actin filaments and bundles, by extending the use of a recently developed form-finding model. The model provides a new approach to building up cytoskeletal computational models and captures the features of cellular 


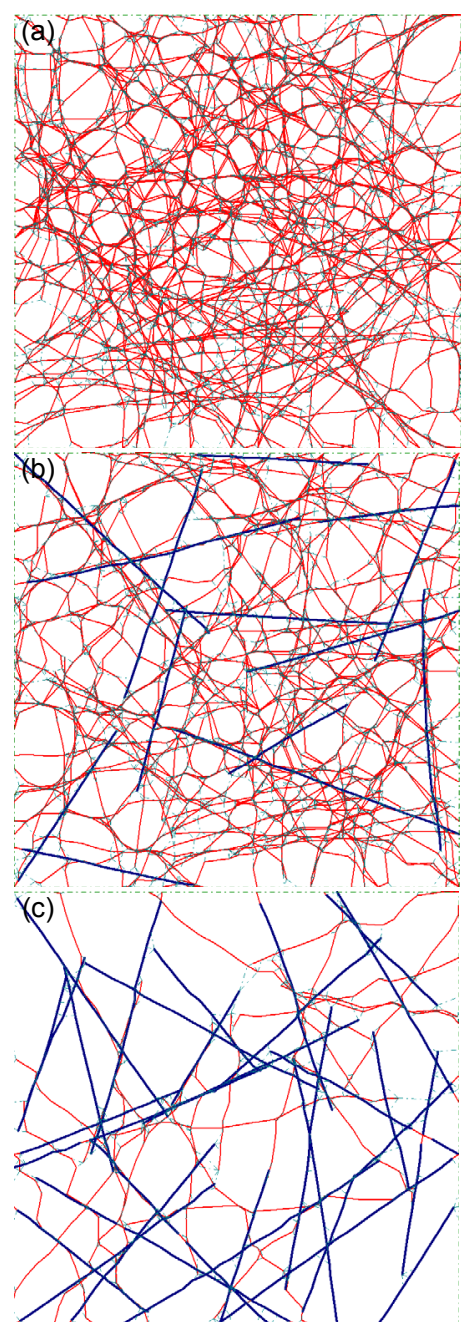

Fig. 9 Layouts of selected samples with different bundle relative densities of (a) $0 \%$, (b) $40 \%$, and (c) $90 \%$ The spread in bundle orientation was $90^{\circ}$, i.e., the bundles were uniformly and randomly distributed in each model. The maximum offset was $0.3 \mu \mathrm{m}$. The color lines are the same as that shown in Fig. 2

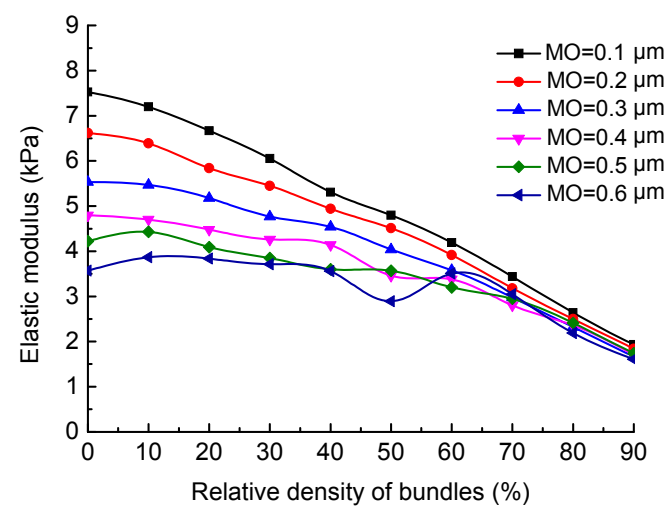

Fig. 10 Variation in the average stiffness of networks with the relative density of bundles, with different values of maximum offset (MO)

The spread in bundle orientation was $90^{\circ}$ in each model mechanical responses in various contexts. The results in this study provide an insight into understanding the complex mechanical performance of cytoskeleton networks of actin filaments and bundles.

The computational model of a cytoskeleton network used in this study contains actin filaments cross-linked and bundled by actin-binding proteins. The effects on network stiffness caused by other cytoskeletal components like microtubules or intermediate filaments were neglected. Also, another assumption in this study was that each actin bundle is modeled as a composite structure of seven actin filaments. In fact, the actin bundle is formed by actin filaments bundled by repeating units of bundling proteins, and the spacing between bundling proteins along the bundle can change over time. Therefore, the bundle is not a composite structure of seven actin filaments and the moment of inertia of bundles calculated in this model is the upper bound. Nevertheless, this is probably not critical to the study of the variation in cytoskeleton network stiffness with the relative density and alignment of bundles.

All the results in this work can be explained by the deformation of the cytoskeleton network model being bending dominated (non-affine) or axial stretching dominated (affine). In the actin networks form-finding model, both bending and axial deformations occur within actin filaments and bundles. The axial stretching stiffness (represented by $E A$, where $E$ is Young's modulus and $A$ is the cross-sectional area of an actin filament or a bundle) is much larger than the bending stiffness (represented by $E I$, where $I$ is the moment of inertia of an actin filament or a bundle) for both filaments and bundles. The quantity $\mathrm{lb}=E I /(E A)$ was used to quantify the relative stiffness of bending and axial deformation. Note that $\mathrm{lb}$ has units of length which, for cylindrical fibers, is equal to half of the cylinder radius (Picu, 2011). For actin filaments, lb is around $0.0035 \mu \mathrm{m}$, and for bundles, around $0.0105 \mu \mathrm{m}$. So an actin network undergoes stiffening in the transition from a bending dominated response to an axial stretching dominated response. It is well known that in a triangle or pattern composed of triangles, deformation under loads is usually axial stretching dominated. However, in a quadrilateral or a polygon, or a pattern composed of quadrilaterals or polygons, deformation under loads is usually bending dominated. So in general, the more filament/bundle 
segments and cross-linkers in an actin model, the easier it is to form more triangles in the layout, and for its deformation trend to be axial stretching dominated, resulting in a stiffer actin network. On the other hand, if an actin filament or a bundle is parallel to the stretching direction, its deformation definitely should be axial stretching dominated. If more actin filaments and bundles in an actin model are parallel to the stretching direction, its deformation should tend to be axial stretching dominated, and the actin network should be stiffer.

Filament undulations cause transverse bending in filaments and bundles, increasing the number of filaments/bundles whose deformation is bending dominated. This is the main reason for the reduction in cytoskeleton network stiffness when filament undulations increase (Fig. 6). The elastic modulus decreases by as much as $50 \%$ in low bundle relative densities (such as $0 \%$ ), but remains almost unchanged in high bundle relative densities $(60 \%$ or $80 \%)$. The reason is that the bending stiffness of filaments is greatly enhanced when they form bundles, and bundles keep almost straight after form-finding. Therefore, more bundles in the actin network make it less sensitive to filament undulations, and the softening effect of filament undulations on network stiffness is not obvious when there is a high relative density of bundles.

The effects of spread in bundle orientation on the stiffness of the cytoskeleton network (Fig. 4) can be explained in a similar way. The bundles generate primarily axial deformation if they align along the stretching direction. On the contrary, if the bundles distribute randomly, only the small number of bundles nearly parallel to the stretching direction will show axial stretching dominated deformation and most others will show bending dominated deformation. As a result, an actin network tends to have an axial stretching dominated response when the angle of all bundles is equal to $0^{\circ}$. However, its deformation tends to show a bending-dominated response when the spread in bundle orientation is $90^{\circ}$. Therefore, the network becomes weaker when the spread in bundle orientation increases from $0^{\circ}$ to $90^{\circ}$.

In this work, the relative density of filaments was fixed at $0.3 \%$ in every model, i.e., the total amount of actin material was constant. Thus, the number of individual filaments was negatively cor- related to the relative density of bundles. On condition that the bundles are distributed randomly in the network, as actin filaments were expended to generate bundles, the total number of segments of actin filaments and bundles, as well as cross-linkers, decreased, and the deformation of actin networks changed from axial stretching dominated (affine) to bending dominated (nonaffine). As a result, the networks became more and more compliant. In contrast, when bundles aligned along the stretching direction, they were dominated mainly by axial deformation. Therefore, as the relative density of bundles increased, the cytoskeleton network became stiffer. However, as most of the actin filaments were expended to generate the parallel bundles, the density of cross-linkers decreased sharply (Fig. 11), weakening the network. This is because the parallel bundles have to be anchored by cross-linkers to provide stiffness for actin networks. The network stiffness peaked when the relative density of bundles was in the range of $30 \%$ to $50 \%$ (Fig. 8 ).

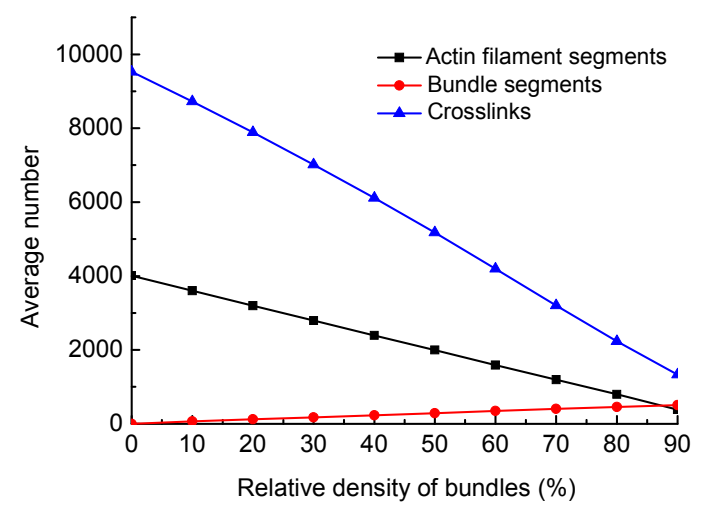

Fig. 11 Variation in the average number of components with the relative density of bundles: the angle of all bundles was $0^{\circ}$ and the maximum offset $0.5 \mu \mathrm{m}$

\section{Conclusions}

In this paper, we have described a mechanical study of a cytoskeleton network consisting of actin filaments and bundles, using a form-finding model. The effects of filament undulations, alignment, and relative density of the bundles on the stiffness of a cytoskeleton network were investigated. The results show that undulations can soften the network by increasing the amount of bending-dominated 
deformations in the model. Parallel bundles along the stretching direction can significantly enhance the network stiffness, while bundles distributed randomly make no contribution to the stiffness. It might be an evolutionary advantage for cells to create parallel bundles rather than disordered bundles during cell adhesion and cell motion. Moreover, with a constant amount of actin filament material, increasing the number of parallel bundles does not invariably increase the stiffness of the cytoskeleton network. The stiffest network contains both actin filaments and bundles.

\section{Acknowledgements}

We are grateful to Prof. Benjamin W. SCHAFER (Department of Civil Engineering, Johns Hopkins University, USA) for the improvement of the form-finding model and enlightening discussions.

\section{References}

Coughlin, M.F., Stamenovic, D., 2003. A prestressed cable network model of the adherent cell cytoskeleton. Biophysical Journal, 84(2):1328-1336. [doi:10.1016/S00063495(03)74948-0]

Ethier, C.R., Simmons, C.A., 2007. Introductory Biomechanics: from Cells to Organisms. Cambridge University Press. [doi:10.1017/CBO9780511809217]

Ferrer, J.M., Lee, H., Chen, J., et al., 2008. Measuring molecular rupture forces between single actin filaments and actin-binding proteins. Proceedings of the National Academy of Sciences, USA, 105(27):9221. [doi:10.1073/ pnas.0706124105]

Furuike, S., Ito, T., Yamazaki, M., 2001. Mechanical unfolding of single filamin A (ABP-280) molecules detected by atomic force microscopy. FEBS Letter, 498(1): 72-75. [doi:10.1016/S0014-5793(01)02497-8]

Gardel, M., Shin, J., MacKintosh, F., et al., 2004. Elastic behavior of cross-linked and bundled actin networks. Science, 304(5675):1301-1305. [doi:10.1126/science. 1095087]

Gong, J.H., Zhang, D.X., Tseng, Y., et al., 2013. Form-finding model shows how cytoskeleton network stiffness is realized. PLOS ONE, 8(10):e77417. [doi:10.1371/journal. pone.0077417]

Huisman, E.M., van Dillen, T., Onck, P.R., et al., 2007. Three-dimensional cross-linked F-actin networks: relation between network architecture and mechanical behavior. Physical Review Letters, 99(20):208103. [doi:10.1103/ PhysRevLett.99.208103]

Kang, J., Steward, R.L., Kim, Y., et al., 2011. Response of an actin filament network model under cyclic stretching through a coarse grained Monte Carlo approach. Journal of Theoretical Biology, 274(1):109-119. [doi:10.1016/ j.jtbi.2011.01.011]

Kasza, K., Broedersz, C., Koenderink, G., et al., 2010. Actin filament length tunes elasticity of flexibly cross-linked actin networks. Biophysical Journal, 99(4):1091-1100. [doi:10.1016/j.bpj.2010.06.025]

Kim, T., Hwang, W., Kamm, R., 2009a. Computational analysis of a cross-linked actin-like network. Experimental Mechanics, 49(1):91-104. [doi:10.1007/s11340-0079091-3]

Kim, T., Hwang, W., Lee, H., et al., 2009b. Computational analysis of viscoelastic properties of crosslinked actin networks. PLoS Computational Biology, 5(7):e1000439. [doi:10.1371/journal.pcbi.1000439]

Lim, C.T., Zhou, E.H., Quek, S.T., 2006. Mechanical models for living cells-a review. Journal of Biomechanics, 39(2):195-216. [doi:10.1016/j.jbiomech.2004.12.008]

Lin, Y.C., Broedersz, C.P., Rowat, A.C., et al., 2010. Divalent cations crosslink vimentin intermediate filament tail domains to regulate network mechanics. Journal of Molecular Biology, 399(4):637-644. [doi:10.1016/j.jmb. 2010.04.054]

Matsushita, S., Adachi, T., Inoue, Y., et al., 2010. Evaluation of extensional and torsional stiffness of single actin filaments by molecular dynamics analysis. Journal of Biomechanics, 43(16):3162-3167. [doi:10.1016/j.jbiomech.2010.07.022]

Onck, P., Koeman, T., van Dillen, T., et al., 2005. Alternative explanation of stiffening in cross-linked semiflexible networks. Physical Review Letters, 95(17):178102. [doi:10.1103/PhysRevLett.95.178102]

Picu, R.C., 2011. Mechanics of random fiber networks-a review. Soft Matter, 7(15):6768-6785. [doi:10.1039/ c1sm05022b]

Satcher, R.L.Jr., Dewey, C.F.Jr., 1996. Theoretical estimates of mechanical properties of the endothelial cell cytoskeleton. Biophysical Journal, 71(1):109-118. [doi:10.1016/S0006-3495(96)79206-8]

Shin, J.H., Mahadevan, L., So, P., et al., 2004. Bending stiffness of a crystalline actin bundle. Journal of Molecular Biology, 337(2):255-261. [doi:10.1016/j.jmb.2004.01. 028]

Stamenovic, D., Coughlin, M.F., 2000. A quantitative model of cellular elasticity based on tensegrity. Journal of Biomechanical Engineering, 122(1):39. [doi:10.1115/1. 429631]

Stamenovic, D., Ingber, D.E., 2002. Models of cytoskeletal mechanics of adherent cells. Biomechanics and Modeling in Mechanobiology, 1(1):95-108. [doi:10.1007/s10237002-0009-9]

Stricker, J., Falzone, T., Gardel, M.L., 2010. Mechanics of the F-actin cytoskeleton. Journal of Biomechanics, 43(1): 9-14. [doi:10.1016/j.jbiomech.2009.09.003]

Tseng, Y., Schafer, B.W., Almo, S.C., et al., 2002. Functional synergy of actin filament cross-linking proteins. Journal 
of Biological Chemistry, 277(28):25609-25616. [doi:10. 1074/jbc.M202609200]

Tseng, Y., Kole, T.P., Lee, J.S.H., et al., 2005. How actin crosslinking and bundling proteins cooperate to generate an enhanced cell mechanical response. Biochemical and Biophysical Research Communications, 334(1):183-192. [doi:10.1016/j.bbrc.2005.05.205]

\section{中文概要：}

\section{本文题目: 基于找形模型研究微丝束对细胞骨架刚度的影响}

Using a form-finding model to analyze the effect of actin bundles on the stiffness of a cytoskeleton network

研究目的：基于找形分析建立的细胞骨架力学模型研究微丝束对细胞骨架刚度的影响。

创新要点: 目前存在的细胞模型很少考虑微丝束对细胞力学特性的重要作用。本文基于细胞找形模型模 拟了同时包含微丝和微丝束的细胞骨架网络结构, 并且分析了细胞中微丝束的排列方向、微 丝束的含量以及微丝波动对细胞刚度的影响。

研究方法: 基于找形模型, 随机生成由微丝、微丝束 (梁单元) 以及交联蛋白 (索单元) 形成的细胞骨 架网络结构, 依靠非线性有限元计算和样本统计, 计算出模型的弹性模量。通过分别改变模 型中微丝束的排列方向、微丝束的含量以及模型初始最大位移等参数, 得出细胞骨架模型的 弹性模量随这些参数的变化趋势, 以此来研究微丝束对细胞刚度的影响。

重要结论: 细胞骨架网络中微丝的波动会导致细胞刚度降低; 与拉伸方向平行排列的微丝束可以显著地 提高细胞的刚度, 相比之下随机分布的微丝束对细胞刚度没有贡献; 在微丝材料总量固定的 情况下, 细胞刚度随着平行排列微丝束含量的增加呈现出先升高后降低的趋势。

关键词组: 细胞骨架网络; 找形模型; 弹性模量; 微丝束 